\title{
Anti-inflammatory effects of flap and lymph node transfer
}

\section{Viitanen, Tiina P.}

2015-12

Viitanen , T P , Visuri , M T , Sulo , E , Saarikko , A M \& Hartiala , P 2015 , '

Anti-inflammatory effects of flap and lymph node transfer ' , Journal of Surgical Research , vol. 199 , no. 2 , pp. 718-725 . https://doi.org/10.1016/j.jss.2015.04.041

http://hdl.handle.net/10138/159086

https://doi.org/10.1016/j.jss.2015.04.041

publishedVersion

Downloaded from Helda, University of Helsinki institutional repository.

This is an electronic reprint of the original article.

This reprint may differ from the original in pagination and typographic detail.

Please cite the original version. 


\title{
Anti-inflammatory effects of flap and lymph node transfer
}

\author{
Tiina P. Viitanen, $\mathrm{MD}, \mathrm{PhD},{ }^{a, 1}$ Mikko T. Visuri, $\mathrm{MD},{ }^{a, 1}$ Eeva Sulo, $\mathrm{MB},{ }^{a}$ \\ Anne M. Saarikko, MD, PhD, ${ }^{a, b}$ and Pauliina Hartiala, MD, PhD ${ }^{a, c, *}$ \\ ${ }^{a}$ Department of Plastic and General Surgery, Turku University Hospital, Turku, Finland \\ ${ }^{\mathrm{b}}$ Cleft Unit, Department of Plastic Surgery, Helsinki University Hospital, Helsinki, Finland \\ ${ }^{\mathrm{c}}$ Department of Medical Microbiology and Immunology, University of Turku, Turku, Finland
}

\section{A R T I C L E I N F O}

Article history:

Received 24 November 2014

Received in revised form

8 March 2015

Accepted 14 April 2015

Available online 24 April 2015

Keywords:

Lymphedema

Lymphatic surgery

Proinflammatory cytokines

Anti-inflammatory cytokines

IL-10

\begin{abstract}
A B S T R A C T
Background: Transfer of healthy tissue is commonly used in the treatment of complicated wounds and in reconstruction of tissue defects. Recently, microvascular lymph node transfer (LN) has been used to improve the lymphatic function in lymphedema patients. To elucidate the biological effects of flap transfer (with and without lymph nodes), we have studied the postoperative production of proinflammatory, anti-inflammatory, prolymphangiogenic and antilymphangiogenic cytokines, and growth factors (interleukin $1 \alpha$ [IL-1 $\alpha$ ], IL-1 $\beta$, tumor necrosis factor $\alpha$ [TNF- $\alpha$ ], IL-10, transforming growth factor $\beta 1$ [TGF- $\beta 1$ ], IL-4 and IL-13, and vascular endothelial growth factor C [VEGF-C] and VEGF-D) in postoperative wound exudate samples.

Methods: Axillary wound exudate samples were analyzed from four patient groups: axillary lymph node dissection (ALND), microvascular breast reconstruction (BR), LN, and combined LN and BR (LN-BR).

Results: The concentration of proinflammatory cytokines was low in all the flap transfer groups as opposed to the ALND group, which showed an extensive proinflammatory response. The level of anti-inflammatory and antifibrotic cytokine IL-10 was increased in the LN-BR group samples compared with the ALND and BR groups. In the LN and LN-BR groups, the cytokine profile showed an anti-inflammatory response.

Conclusions: Transfer of healthy tissue hinders the proinflammatory response after surgery, which may explain the beneficial effects of flap transfer in various patient groups. In addition, flap transfer with lymph nodes seems to also promote an antifibrotic effect. The clinical effects of LN in lymphedema patients may be mediated by the increased production of prolymphangiogenic growth factor (VEGF-C) and antifibrotic cytokine (IL-10).
\end{abstract}

(c) 2015 Elsevier Inc. All rights reserved.

\section{Introduction}

Surgery and trauma lead to tissue injury and wound healing, which in the early stages is dominated by a proinflammatory response, followed by proliferative and remodeling phases. The inflammation can persist abnormally in the presence of extensive trauma, insufficient circulation, infection or inflammatory disorders, resulting in abnormally healing

\footnotetext{
* Corresponding author. Department of Plastic and General Surgery, Turku University Hospital, P.O. Box 52, Kiinamyllynkatu 4-8, 20251 Turku, Finland. Tel.: +358 2 3131215; fax: +358 23132215.

E-mail address: pauliina.hartiala@tyks.fi (P. Hartiala).

${ }^{1}$ Equal contribution. 0022-4804/\$ - see front matter ( 2015 Elsevier Inc. All rights reserved.
} 
wounds, unwanted scarring, and, in some patients, also lymphedema. Transfer of healthy tissue is commonly used in the treatment of complicated and chronic wounds [1,2]. Few studies have evaluated the inflammatory effects of flap transfer surgery used in breast reconstruction (BR). Microvascular BR has been shown to result in a greater systemic elevation of the acute phase inflammatory $C$ reactive protein [3] and interleukin 6 (IL-6) [4] compared with local pedicular latissimus dorsi or lateral thoracodorsal BR flaps. However, both of these studies evaluated only the systemic response of surgery, and comparison to nonflap surgery was not performed. Thus far, little is known about the effects of flap transfer on the local wound healing environment.

More recently, autologous microvascular lymph node transfer (LN) has been used to improve the lymphatic drainage in lymphedema patients [5-7]. In addition to restoring the lymphatic flow, LN offers a possibility to retain the lymphatic, immunologic, and sentinel node functions of the affected limb. Interestingly, also flap transfer without lymph nodes (normal BR) has been shown to reduce the lymphedema symptoms of the arm in some postsurgical lymphedema patients [8-10]. This suggests that in some cases flap transfer alone modifies the local wound environment favorable to lymphangiogenesis.

Human lymph nodes express lymphatic vascular endothelial growth factor C (VEGF-C) [6], which is secreted into axillary wound exudate after microvascular LN [7]. Results from the preclinical lymphedema models using VEGF-C or VEGF-D have demonstrated the ability of these factors to induce the growth of new lymphatic vessels [11-13], thus providing a biological basis for the microsurgical LN method.
Recent studies have shown that in addition to VEGF-C, lymphangiogenesis is regulated by a coordinated expression of proinflammatory and anti-inflammatory cytokines [14-16]. Furthermore, fibrosis and scarring are known to be key inhibitors of lymphatic regeneration [17]. Therefore, the factors affecting chronic inflammation and fibrosis are also a major topic of interest.

The clinical benefits of flap transfer surgery in various wound healing disorders are acknowledged [1,2], although there is lack of supportive biological evidence. To provide more information about the local biological and immunologic effects of flap transfer with and without lymph nodes, we decided to evaluate the postoperative production of proinflammatory cytokines (IL-1 $\alpha$, IL-1 $\beta$, and tumor necrosis factor alpha [TNF- $\alpha]$ ), anti-inflammatory cytokines (IL-10 and transforming growth factor beta1 [TGF- $\beta 1]$ ), profibrotic cytokines (IL-4 and IL-13), and lymphatic growth factors (VEGF-C and $-D$ ) involved in wound healing and lymphangiogenesis in different patient groups: axillary lymph node dissection (ALND), microvascular BR, LN, and combined LN and BR (LNBR; study design in Fig. 1).

\section{Patients and methods}

\subsection{Patient samples for cytokine analysis}

Permission for collecting patient samples was approved by the Ethical Committee of the Turku University Hospital. All patients signed an approval for sample collection and approved the use of their patient information in the study. Postoperative

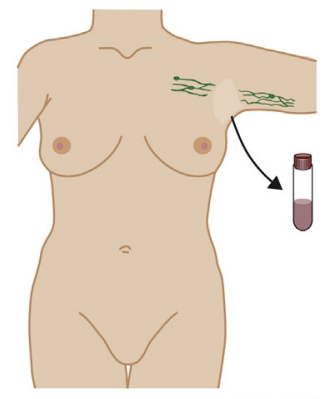

Axillary lymph node removal (ALND)

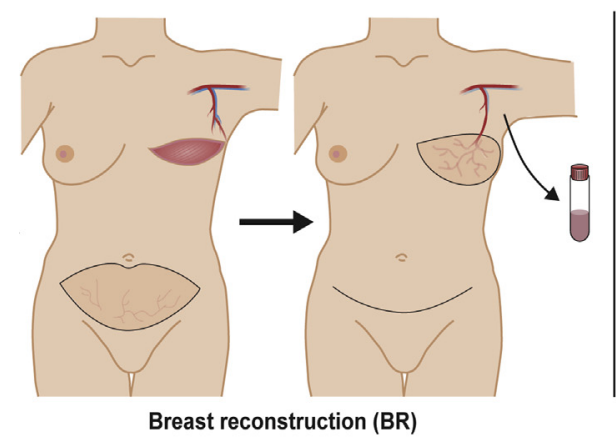

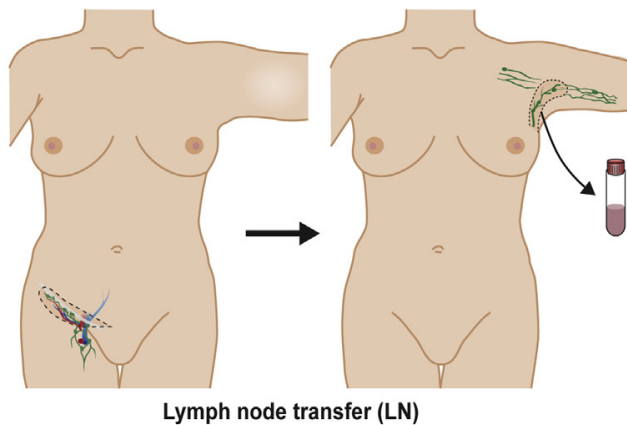

Lymph node transfer (LN)

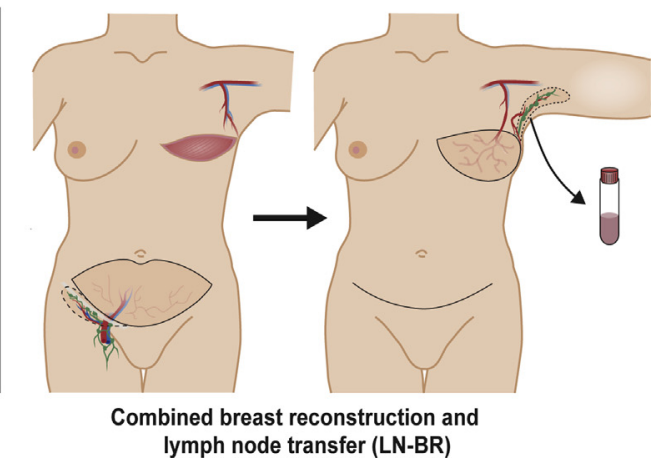

Fig. 1 - Patient groups for wound exudate samples are as follows: ALND, LN, BR, and LN-BR. Cytokines and growth factors

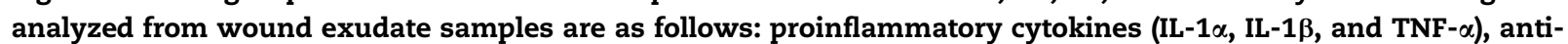
inflammatory cytokines (IL-10 and TGF-ק1), profibrotic cytokines (IL-4, IL-13), and lymphatic growth factors (VEGF-C and -D). (Color version of figure is available online.) 
axillary wound exudate samples were collected on the first and sixth postoperative day (POD) from LN $(n=8)$, LN-BR $(n=8)$ and, for controls, BR $(n=8)$ and ALND $(n=8)$ patients. In addition, wound exudate samples were collected from flap donor sites $(n=9)$ on the first and sixth POD. Microvascular LN was performed to lymphedema patients as previously described [6,7]. The BR group consisted of postmastectomy patients without lymphedema symptoms. Preparation of the axilla was similar in all the flap groups (LN, $\mathrm{LN}-\mathrm{BR}$, and $\mathrm{BR}$ ) as the thoracodorsal vessels were used as recipient vessels in all the $B R$ flaps and the retrograde thoracodorsal vessels in the lymphatic flaps. ALND patients underwent a routine breast cancer operation (mastectomy or breast resection) with removal of axillary lymph nodes due to metastases. The decision for ALND was made preoperatively based on imaging and biopsy results, and thus sentinel node biopsy was not performed. Exclusion criteria in the ALND group were preoperative oncological treatments or previous axillary surgery.

\subsection{Evaluation of wound exudate cytokines}

A protease inhibitor (cOmplete EDTA-free Protease Inhibitor Cocktail Tablets; Roche Diagnostics, Mannheim, Germany) was added to the wound exudate samples, followed by centrifugation to separate the supernatant and cell pellet [7]. Supernatant cytokine protein concentrations were measured using enzyme-linked immunosorbent assays (R\&D Systems, Minneapolis, MN) according to the manufacturer's instructions. Optical densities were read with a microplate reader (Infinite 200; Tecan Group Ltd, Männedorf, Switzerland) and converted to picograms per milliliter with MasterPlex ReaderFit 2.0.0.77 (MiraiBio Group of Hitachi Solutions America, Ltd, San Francisco, CA).

\subsection{Statistical analyses}

All tests were performed two tailed and P-values $<0.05$ considered statistically significant. Differences between the treatment groups were tested separately on the first and sixth POD, using either parametric (analysis of variance) or nonparametric (Kruskal-Wallis) one-way analysis of variance, and when appropriate, followed by pairwise comparisons tests.
For normally distributed data, effect sizes for pairwise comparisons are reported as Hedges' $g$ statistic with a 95\% confidence interval (CI), whereas Cliff's delta statistic with a 95\% CI is used for nonnormally distributed data. Results are expressed as arithmetic means ( \pm standard deviation) for normally distributed data (Figs. 3,5,7A and B and Supplementary Figs. $4,6 \mathrm{~B}, 7,8)$ and geometric means (95\% CIs) for log-transformed data (Figs. 2,4,6 and Supplementary Figs. 1A and B,2,3A and $B, 5,6 \mathrm{~A})$, with error bars in the respective figures representing $95 \%$ CIs. For nonnormally distributed and nontransformed data (Fig. 5 and Supplementary Fig. 7), results are reported as medians (interquartile ranges), with horizontal lines in the graphs indicating medians, boxes interquartile ranges, and whiskers extending from minimum to maximum values. A detailed description of the statistical analyses can be found in the supplementary data files.

\section{3. \\ 3.1. Proinflammatory cytokines IL-1 $\alpha$, IL-1 $\beta$, and TNF- $\alpha$}

Analysis of the cytokine concentrations revealed only nonsignificant and nonsubstantial differences in the IL- $1 \alpha$ levels between any of the groups on the first or sixth POD (Supplementary Fig. 1A and B, Supplementary Table 1).

As for the IL-1 $\beta$ concentrations on the first POD (Fig. 2, Supplementary Table 2), the levels were significantly higher in the ALND group $(P=0.001)$ and nonsignificantly but still substantially higher also in the LN-BR group $(P=0.066$, $\mathrm{g}=1.38,95 \% \mathrm{CI}=0.29-2.47)$, when compared to the BR group. For the rest of the pairwise comparisons, the differences were nonsignificant and nonsubstantial. On the sixth POD (Supplementary Fig. 2, Supplementary Table 2), the IL-1 $\beta$ concentrations no longer differed significantly or substantially between the groups.

In terms of TNF- $\alpha$, only nonsignificant and nonsubstantial differences between the groups were observed on the first POD (Supplementary Fig. 3A, Supplementary Table 3). However, on the sixth POD (Supplementary Fig. 3B, Supplementary Table 3), even though the groupwise differences were nonsignificant, the level of TNF- $\alpha$ in the ALND group was still substantially higher than that of the LN-BR group $(P=0.052$,

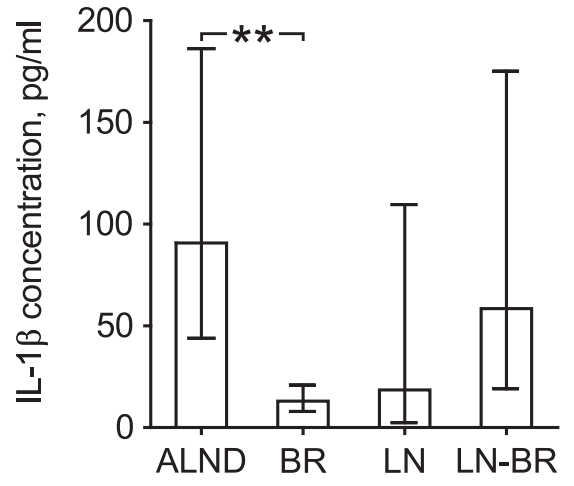

1st POD

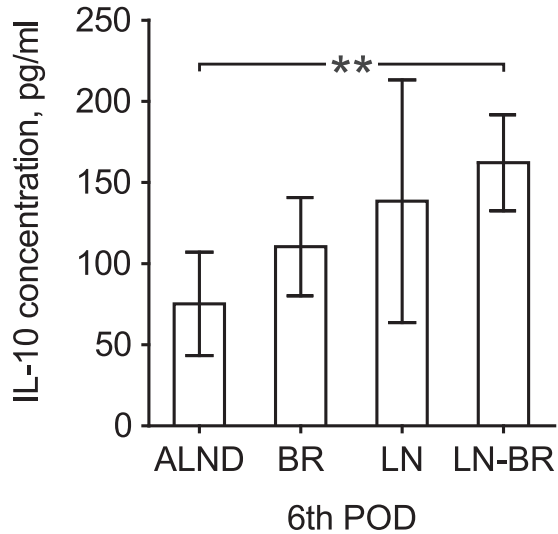

Fig. 3 - Immunoregulatory cytokine IL-10. ${ }^{* *} P<0.01$.

Fig. 2 - Proinflammatory cytokine IL-1 $\beta{ }^{* *} \mathrm{P}<0.01$. 


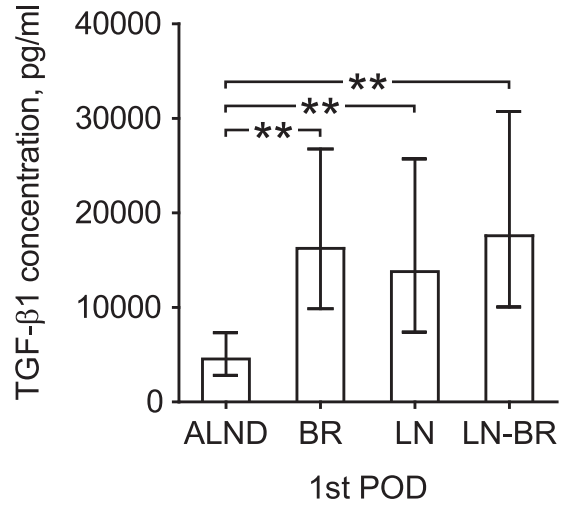

Fig. 4 - Transforming growth factor $\beta 1 .{ }^{* *} P<0.01$.

$\mathrm{g}=1.44,95 \% \mathrm{CI}=0.34-2.54)$. No other substantial differences were found.

\subsection{Anti-inflammatory cytokines IL-10 and TGF- $\beta 1$}

In terms of IL-10, the groupwise differences in the concentrations turned out nonsignificant and nonsubstantial on the first POD (Supplementary Fig. 4, Supplementary Table 4). On the sixth POD, the IL-10 concentrations were higher in the LN groups, LN and LN-BR, when compared to the control groups, ALND and BR (Fig. 3, Supplementary Table 4). Although this difference was significant only between LN-BR and ALND $(P=0.004)$, the comparison between LN-BR and BR proved still substantial $(P=0.165, g=1.36,95 \% C I=0.28-2.45)$. As for the rest of the groupwise comparisons, the results were nonsignificant and nonsubstantial.

To determine whether the differences between the LN (LN and LN-BR) and the BR control groups were due to the transferred lymph nodes or possibly other characteristics of the patient groups (e.g., lymphedema versus nonlymphedema), the IL-10 concentrations were measured also from flap donor site wound exudate. However, no significant or substantial differences were found between the flap transfer groups either on the first or sixth POD (data not shown).

As for TGF- $\beta 1$, the protein levels on the first POD (Fig. 4, Supplementary Table 5) were significantly higher in all other

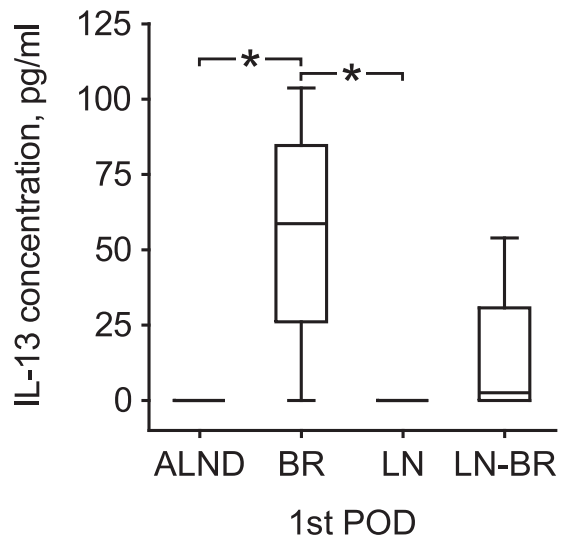

Fig. 5 - Profibrotic cytokine IL-13. ${ }^{*} P<0.05$.

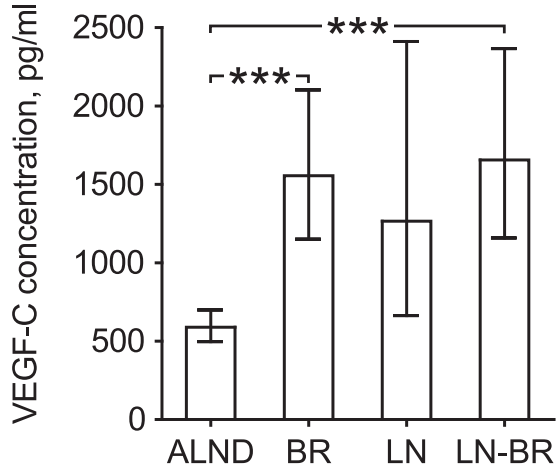

1st POD

Fig. 6 - Vascular endothelial growth factor C. ${ }^{* * *} \mathrm{P}<0.001$. Preliminary VEGF-C results with smaller number of samples have been published in our earlier report [7].

groups when compared with ALND: ALND versus BR, $P=0.003$; ALND versus LN, $P=0.010$; and ALND versus LN-BR, $P=0.001$. Among these flap transfer groups, the TGF- $\beta 1$ concentrations were similar and the differences were nonsignificant and nonsubstantial. In contrast to the first POD, the levels on the sixth POD were low also in the flap transfer groups and no significant or substantial differences were observed (Supplementary Fig. 5, Supplementary Table 5).

\subsection{Profibrotic cytokines IL-4 and IL-13}

Analysis of the exudate samples revealed no significant or substantial differences in the IL-4 levels between any of the groups on the first or sixth POD (Supplementary Fig. 6A and B, Supplementary Table 6).

On the other hand, more prominent groupwise differences were observed in the IL-13 concentration on the first POD (Fig. 5, Supplementary Table 7). However, only comparisons of BR with ALND and LN were significant, even though the level of IL-13 was virtually zero in the ALND and LN groups and markedly higher in the BR and LN-BR groups: BR versus ALND, $P=0.029$; $B R$ versus $L N, P=0.029$. The rest of the groupwise differences were nonsignificant and nonsubstantial. On the sixth POD, no significant or substantial differences were observed (Supplementary Fig. 7, Supplementary Table 7).
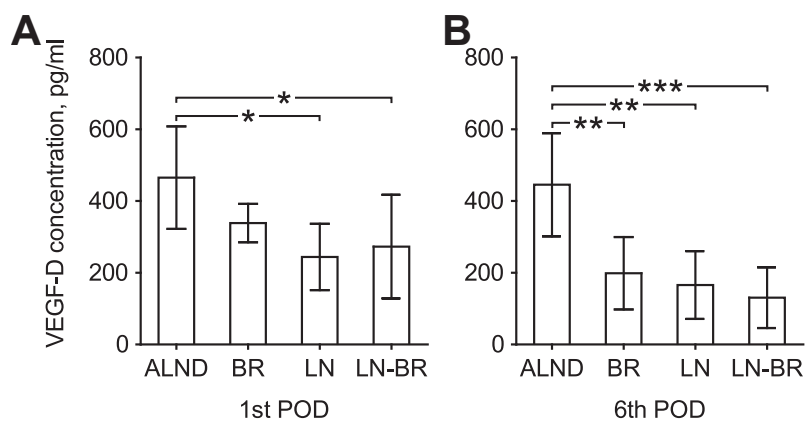

Fig. 7 - Vascular endothelial growth factor $D .{ }^{*} P<0.05$; ${ }^{* *} \mathrm{P}<0.01 ;{ }^{* * *} \mathrm{P}<0.001$. 


\subsection{Lymphangiogenic growth factors VEGF-C and VEGF-D}

Similar to our previous study with a smaller number of patients [7], significant groupwise differences in the VEGF-C concentration were observed on the first POD (Fig. 6, Supplementary Table 8). Compared with ALND, the level of VEGF-C was significantly higher in the BR $(P<0.001)$ and LNBR $(P<0.001)$ groups, and in addition, nonsignificantly but still substantially higher also in the LN group $(P=0.099$, $\mathrm{g}=1.51,95 \% \mathrm{CI}=0.42-2.61)$. The differences between these three groups were nonsignificant and nonsubstantial. On the sixth POD, the VEGF-C concentrations in the flap groups were similar to ALND and no significant or substantial differences were found (Supplementary Fig. 8, Supplementary Table 8).

Interestingly, the results from the VEGF-D level analysis were somewhat reciprocal. On the first POD, the level of VEGF$D$ was highest in the ALND group, significantly higher when compared with the LN groups: ALND versus LN, $P=0.017$; ALND versus LN-BR, $P=0.047$ (Fig. 7A, Supplementary Table 9). On the sixth POD, the situation was further emphasized (Fig. 7B, Supplementary Table 9) and all comparisons with the ALND group were now significant: ALND versus $B R, P=0.004$; ALND versus $L N, P=0.001$; and ALND versus $L N-B R, P<0.001$. As for the rest of the groupwise comparisons of both PODs, only nonsignificant and nonsubstantial differences were observed.

\subsection{Wound exudate cytokine profiles}

To summarize the results, groupwise cytokine profiles were generated from the relative concentrations (groupwise mean divided by the grand mean; Fig. $8 \mathrm{~A}$ and B). On the first POD, the level of proinflammatory cytokines was notably high for the ALND group and low for the BR group. The level of profibrotic cytokines was, in contrast, low for the ALND group and high for the BR group, particularly with IL-13. As for the anti-inflammatory cytokine concentrations, the differences between the ALND and BR groups were less prominent. The results on the sixth POD were similar to those of the first POD, with the exception of TNF- $\alpha$, now somewhat elevated also in the BR group. Interestingly, in the LN groups, cytokine profiles were less distinct on both PODs, as the relative concentrations were mainly quite similar to the grand mean, the most persistent exceptions being the low TNF- $\alpha$ and IL-13 levels of the LN group on both the first and sixth POD, and the elevated IL-10 levels on the sixth POD in the LN-BR and, to a smaller degree also in the LN group.

\section{Discussion}

In this study, we investigated postoperative axillary exudate samples from four patient groups (ALND, BR, LN, and LN-BR; Fig. 1). Our results showed that the concentration of antiinflammatory cytokines was increased after flap transfer, whereas in the ALND patients the proinflammatory cytokines dominated the profile. In addition, flap transfer with lymph nodes seemed to promote also an antifibrotic response (Figs. 8 and 9). Furthermore, we observed unexpected differences in the VEGF-C and VEGF-D production profiles (summary in Fig. 9). This is to our knowledge the first study investigating the inflammatory effects of LN surgery and one of the few studies elucidating the local biological mechanisms of flap transfer.

\subsection{Transfer of healthy tissue may reduce the local proinflammatory response}

Inflammation seems to play a diversified role in wound healing and the development of lymphedema. Several studies have demonstrated that inflammation is closely related to lymphangiogenesis, and proinflammatory cytokines induce lymphangiogenic growth factor (VEGF-C) expression in experimental settings $[14,15]$. On the contrary, lymphatic stasis is known to initiate chronic inflammation and tissue fibrosis resulting in worsening of the lymphatic function [18]. Furthermore, lymphedema has been associated with a similar gene expression profile as inflammation in a mouse model [19]. According to our results, it seems that removal of axillary lymphatic tissue promotes a proinflammatory response, whereas transfer of healthy tissue to the axillary area may reduce this effect.

There seemed to be no significant differences in the concentrations of proinflammatory cytokines between the different flap transfer groups. We suggest that low production of these proinflammatory cytokines is related primarily to the transfer of healthy tissue, rather than the transferred lymph

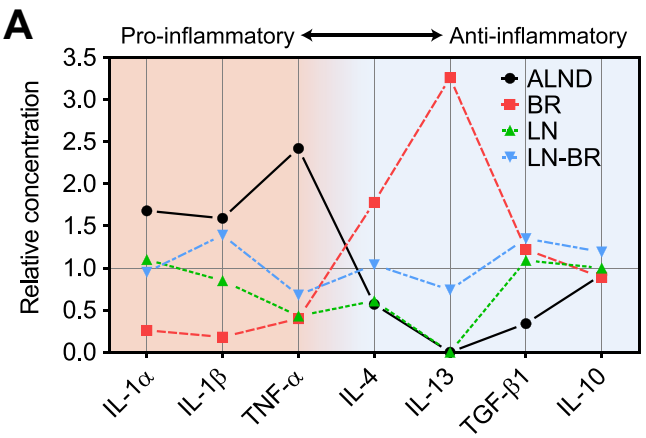

1st POD

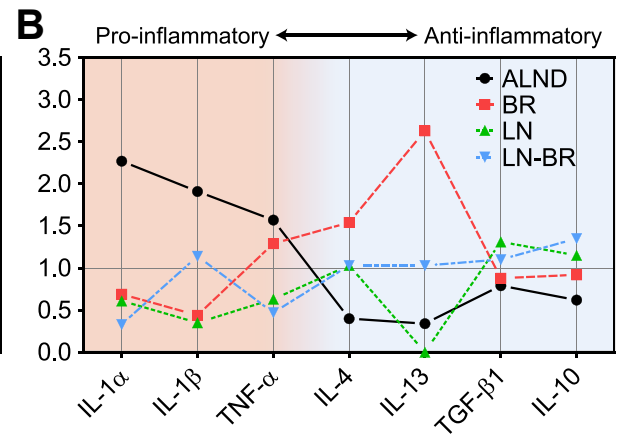

6th POD

Fig. 8 - Summary of the relative cytokine concentrations in the different patient groups. Cytokines are arranged in the figure according to their type: proinflammatory cytokines are located on the left, profibrotic cytokines are in the middle, and antiinflammatory cytokines on the right side of the figure. (Color version of figure is available online.) 


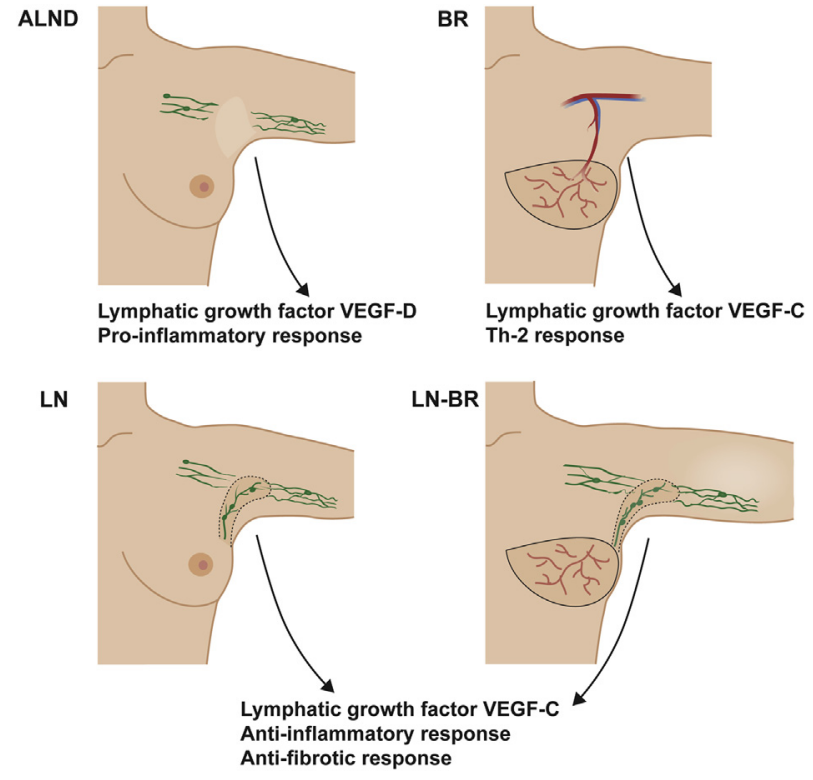

Fig. 9 - Summary of the lymphatic and inflammatory responses in the different patient groups. (Color version of figure is available online.)

nodes. Interestingly, Brown et al. [20] have shown that the transfer of a muscle flap decreases the production of proinflammatory cytokine TNF- $\alpha$ in an experimental fracture model. The transfer of healthy tissue is commonly used in the treatment of infectious wound complications [1,2] and conditions caused by chronic skin inflammation [21]. In addition, according to recent reports, normal BR (without lymph nodes) may improve the lymphatic function of the affected arm in some patients [8,9]. Furthermore, immediate BR seems to reduce the risk of developing lymphedema [10]. It is therefore possible that this clinical finding is partly related to the smaller production of proinflammatory cytokines after flap transfer. However, the production of lymphangiogenic growth factor (VEGF-C) after flap transfer (also without lymph nodes) [7] probably also plays a role in inducing beneficial effects in lymphedema patients (Fig. 9).

\subsection{Role of prolymphangiogenic growth factors VEGF-C and VEGF-D}

Members of the VEGFs family are important regulators of angiogenesis and lymphangiogenesis [22,23]. VEGF-C and VEGF-D both induce lymphangiogenesis by enhancing proliferation, migration, and survival of lymphatic endothelial cells [24]. Human lymph nodes express endogenous VEGF-C, which provides a biological basis for the microsurgical LN method [6]. In our earlier publication, we found an increased concentration of VEGF-C in the axillary wound exudate after flap transfer in the LN and LN-BR groups and, surprisingly, also in the BR group, compared with the ALND group [7]. This finding was confirmed with a greater number of patient samples in the present study. One explanation for the high VEGF-C concentration in the BR patients might be the finding that patients, who have not developed lymphedema after axillary clearance, have actually residual axillary lymph nodes [25], which are producing VEGF-C. In addition, recruited macrophages have been shown to be the source of VEGF-C after flap transfer [26].

Interestingly, production of another important prolymphangiogenic growth factor, VEGF-D, differed markedly from VEGF-C production after the LN. Even though VEGF-C and $-D$ activate same target receptors in the lymphatic vessel endothelium, they have been reported to have different roles also during the embryonic development and growth $[27,28]$. High VEGF-D concentrations after the axillary dissection may suggest that VEGF-D, rather than VEGF$C$, could play a role in the spontaneous lymphatic vascular regrowth in the axilla after the breast cancer surgery. Furthermore, the ALND patients in this study have undergone axillary dissection because of a breast cancer lymph node metastasis, whereas the patients in the other groups did not have active cancer at the time of surgery. Recently operated carcinoma may alter the local VEGF-D concentration, as some breast cancer types are known to secrete lymphatic growth factors to enhance lymphatic invasion and lymph node metastasis [29-31].

\subsection{LN promotes an anti-inflammatory and antifibrotic response}

The most interesting finding in our study was the fact that the concentration of IL-10 was higher in the LN groups when compared with the BR and ALND groups. IL-10 is an antiinflammatory cytokine produced primarily by regulatory macrophages [32]. The major physiological importance of IL10 is the prevention of uncontrolled harmful immunologic reactions [32]. To our knowledge, the direct effect of IL-10 on lymphangiogenesis has not been studied. However, it has been shown that IL-10 can reduce scar formation and fibrosis by inhibiting excessive deposition of extracellular matrix and by regulating the arrangement of collagen fibers in regenerated tissue [33]. It has also been shown that IL-10 downregulates fibrosis promoting proinflammatory cytokines [34]. In both preclinical and clinical studies, IL-10 has been found to be efficient in scar reduction and scar-improving therapies $[33,35,36]$. In light of these previous studies, it is possible that the clinical effects of LN are partly mediated by the increased production of IL-10, which has anti-inflammatory and also antifibrotic properties. The fact that the LN and LN-BR group patients have lymphedema, whereas the BR and ALND patients do not have, should not affect the results, as we know that immune cells have the ability to react to changing circumstances rather quickly. Therefore on POD 1 and 6, we are most likely to see the effects of radical surgery rather than the prior lymphedema.

TGF- $\beta 1$ is an anti-inflammatory cytokine [37], and also a regulator of tissue fibrosis and scarring in the later stages of wound healing $[38,39]$. It has been demonstrated that inhibition of TGF- $\beta 1$ leads to increased lymphatic repair during wound healing [38]. IL-10 is also known to protect from TGF$\beta 1-$ induced fibrosis [33]. In our material, the TGF- $\beta 1$ concentration in the axillary wound exudate on the first POD was higher in all the flap transfer groups compared with the ALND group, but on the sixth POD the concentration was low in all 
groups. In light of our results and previously published studies, it can be speculated that in the early stages of wound healing, the anti-inflammatory properties of TGF- $\beta 1$ are beneficial, but in the later stages of wound remodeling a high concentration of TGF- $\beta 1$ may inhibit lymphangiogenesis by causing extensive fibrosis.

In addition to TGF- $\beta 1$, also IL-4 and IL-13 have been shown to be profibrotic and contribute to the development of lymphedema [40]. The highest concentrations of these profibrotic cytokines were found in the BR group, although the concentrations were generally quite low. Thus, further evidence is needed to clarify the role of these profibrotic factors in the regulation of lymphangiogenesis in human patients.

\section{Conclusions}

The transfer of healthy tissue is commonly used in the treatment of complicated wounds in various patient groups $[1,2,21]$. Recently, flap transfer with lymph nodes has been used in the treatment of lymphedema patients $[5,6,41,42]$. In clinical studies, the LN seems to improve lymphatic flow of the affected arm [5-7]. In addition, the role of flap transfer without lymph nodes in the prevention and resolution of lymphedema has been speculated recently [8-10]. In this study, we have provided new information about the local immunologic effects of flap transfer. According to our study, flap transfer seems to prevent the proinflammatory response after surgery, providing one explanation for the beneficial effects seen after flap transfer in chronic inflammatory and infectious wounds. Interestingly, LN seems to promote not only a prolymphangiogenic effect, but also an antiinflammatory and antifibrotic response (Fig. 9). The cytokine profile after an LN seems to differ from flap transfer without lymph nodes, suggesting that also the transferred lymph nodes are influencing locally on the wound healing environment.

\section{Acknowledgment}

The authors acknowledge Dr Erkki Suominen, the crucial member and teacher of surgical team. The authors also thank all the nurses and secretaries in the Department of Plastic and General Surgery of Turku University Hospital.

This study was supported by Special Governmental Funding (EVO) allocated to Turku University Hospital and Finnish Cultural Foundation Varsinais Suomi Regional Fund, TYKS Foundation, Emil Aaltonen Foundation, and Ida Montini Foundation.

Authors' contributions: T.P.V., member of surgical team, wrote the article and collected wound exudate samples. M.T.V contributed to the collection and analysis of wound exudate samples, statistics, and writing. E.S. contributed to the collection of wound exudate samples and writing. A.M.S., member of surgical team, did the design of the study and writing of the article. P.H. did the design of the study and writing of the article.

\section{Disclosure}

None.

\section{Supplementary data}

Supplementary data related to this article can be found at http://dx.doi.org/10.1016/j.jss.2015.04.041.

\section{R E F E R E N C E S}

[1] Corten K, Struelens B, Evans B, Graham E, Bourne RB, Macdonald SJ. Gastrocnemius flap reconstruction of softtissue defects following infected total knee replacement. Bone Joint J 2013;95-B:1217.

[2] Cabbabe EB, Cabbabe SW. Immediate versus delayed onestage sternal debridement and pectoralis muscle flap reconstruction of deep sternal wound infections. Plast Reconstr Surg 2009;123:1490.

[3] Blomqvist L, Malm M, Berg A, Svelander L, Kleinau S. The inflammatory reaction in elective flap surgery. Plast Reconstr Surg 1998;101:1524.

[4] Schmidt A, Bengtsson A, Tylman M, Blomqvist L. Proinflammatory cytokines in elective flap surgery. J Surg Res 2007;137:117.

[5] Becker C, Assouad J, Riquet M, Hidden G. Postmastectomy lymphedema: long-term results following microsurgical lymph node transplantation. Ann Surg 2006;243:313.

[6] Saaristo AM, Niemi TS, Viitanen TP, Tervala TV, Hartiala P, Suominen EA. Microvascular breast reconstruction and lymph node transfer for postmastectomy lymphedema patients. Ann Surg 2012;255:468.

[7] Viitanen TP, Visuri MT, Hartiala P, et al. Lymphatic vessel function and lymphatic growth factor secretion after microvascular lymph node transfer in lymphedema patients. Plast Reconstr Surg Glob Open 2013;1.

[8] Abbas Khan MA, Mohan A, Hardwicke J, et al. Objective improvement in upper limb lymphoedema following ipsilateral latissimus dorsi pedicled flap breast reconstruction-a case series and review of literature. J Plast Reconstr Aesthet Surg 2011;64:680.

[9] Blanchard M, Arrault M, Vignes S. Positive impact of delayed breast reconstruction on breast-cancer treatment-related arm lymphoedema. J Plast Reconstr Aesthet Surg 2012;65: 1060.

[10] Card A, Crosby MA, Liu J, Lindstrom WA, Lucci A, Chang DW. Reduced incidence of breast cancer-related lymphedema following mastectomy and breast reconstruction versus mastectomy alone. Plast Reconstr Surg 2012;130:1169.

[11] Saaristo A, Karkkainen MJ, Alitalo K. Insights into the molecular pathogenesis and targeted treatment of lymphedema. Ann N Y Acad Sci 2002;979:94.

[12] Baker A, Kim H, Semple JL, et al. Experimental assessment of pro-lymphangiogenic growth factors in the treatment of post-surgical lymphedema following lymphadenectomy. Breast Cancer Res 2010;12:R70.

[13] Lahteenvuo M, Honkonen K, Tervala T, et al. Growth factor therapy and autologous lymph node transfer in lymphedema. Circulation 2011;123:613.

[14] Alitalo K. The lymphatic vasculature in disease. Nat Med 2011;17:1371. 
[15] Ristimaki A, Narko K, Enholm B, Joukov V, Alitalo K. Proinflammatory cytokines regulate expression of the lymphatic endothelial mitogen vascular endothelial growth factor-C. J Biol Chem 1998;273:8413.

[16] Zampell JC, Avraham T, Yoder N, et al. Lymphatic function is regulated by a coordinated expression of lymphangiogenic and anti-lymphangiogenic cytokines. Am J Physiol Cell Physiol 2012;302:C392.

[17] Avraham T, Clavin NW, Daluvoy SV, et al. Fibrosis is a key inhibitor of lymphatic regeneration. Plast Reconstr Surg 2009;124:438.

[18] Avraham T, Daluvoy S, Zampell J, et al. Blockade of transforming growth factor-beta1 accelerates lymphatic regeneration during wound repair. Am J Pathol 2010;177: 3202.

[19] Tabibiazar R, Cheung L, Han J, et al. Inflammatory manifestations of experimental lymphatic insufficiency. PLoS Med 2006;3:e254.

[20] Brown SA, Mayberry AJ, Mathy JA, Phillips TM, Klitzman B, Levin LS. The effect of muscle flap transposition to the fracture site on TNFalpha levels during fracture healing. Plast Reconstr Surg 2000;105:991.

[21] Busnardo FF, Coltro PS, Olivan MV, Busnardo AP, Ferreira MC. The thoracodorsal artery perforator flap in the treatment of axillary hidradenitis suppurativa: effect on preservation of arm abduction. Plast Reconstr Surg 2011;128:949.

[22] Oliver G. Lymphatic vasculature development. Nat Rev Immunol 2004;4:35.

[23] Lohela M, Bry M, Tammela T, Alitalo K. VEGFs and receptors involved in angiogenesis versus lymphangiogenesis. Curr Opin Cell Biol 2009;21:154.

[24] Tammela T, Enholm B, Alitalo K, Paavonen K. The biology of vascular endothelial growth factors. Cardiovasc Res 2005; 65:550.

[25] Szuba A, Pyszel A, Jedrzejuk D, Janczak D, Andrzejak R. Presence of functional axillary lymph nodes and lymph drainage within arms in women with and without breast cancer-related lymphedema. Lymphology 2007;40:81.

[26] Yan A, Avraham T, Zampell JC, Aschen SZ, Mehrara BJ. Mechanisms of lymphatic regeneration after tissue transfer. PLoS One 2011;6:e17201.

[27] Tammela T, Alitalo K. Lymphangiogenesis: molecular mechanisms and future promise. Cell 2010;140:460.

[28] Olsson AK, Dimberg A, Kreuger J, Claesson-Welsh L. VEGF receptor signalling - in control of vascular function. Nat Rev Mol Cell Biol 2006;7:359.
[29] Achen MG, Jeltsch M, Kukk E, et al. Vascular endothelial growth factor D (VEGF-D) is a ligand for the tyrosine kinases VEGF receptor 2 (Flk1) and VEGF receptor 3 (Flt4). Proc Natl Acad Sci U S A 1998;95:548.

[30] Alitalo K, Tammela T, Petrova TV. Lymphangiogenesis in development and human disease. Nature 2005;438:946.

[31] Tobler NE, Detmar M. Tumor and lymph node lymphangiogenesis-impact on cancer metastasis. J Leukoc Biol 2006;80:691.

[32] Asadullah K, Sterry W, Volk HD. Interleukin-10 therapy-review of a new approach. Pharmacol Rev 2003;55: 241.

[33] Shi JH, Guan H, Shi S, et al. Protection against TGF-beta1induced fibrosis effects of IL-10 on dermal fibroblasts and its potential therapeutics for the reduction of skin scarring. Arch Dermatol Res 2013;305:341.

[34] Singer AJ, Clark RA. Cutaneous wound healing. N Engl J Med 1999;341:738.

[35] Peranteau WH, Zhang L, Muvarak N, et al. IL-10 overexpression decreases inflammatory mediators and promotes regenerative healing in an adult model of scar formation. J Invest Dermatol 2008;128:1852.

[36] Kieran I, Knock A, Bush J, et al. Interleukin-10 reduces scar formation in both animal and human cutaneous wounds: results of two preclinical and phase II randomized control studies. Wound Repair Regen 2013;21:428.

[37] Li X, Mai J, Virtue A, et al. IL-35 is a novel responsive antiinflammatory cytokine - a new system of categorizing antiinflammatory cytokines. PLoS One 2012;7:e33628.

[38] Clavin NW, Avraham T, Fernandez J, et al. TGF-beta1 is a negative regulator of lymphatic regeneration during wound repair. Am J Physiol Heart Circ Physiol 2008;295:H2113.

[39] Penn JW, Grobbelaar AO, Rolfe KJ. The role of the TGF-beta family in wound healing, burns and scarring: a review. Int J Burns Trauma 2012;2:18.

[40] Avraham T, Zampell JC, Yan A, et al. Th2 differentiation is necessary for soft tissue fibrosis and lymphatic dysfunction resulting from lymphedema. FASEB J 2013;27:1114.

[41] Cheng MH, Chen SC, Henry SL, Tan BK, Lin MC, Huang JJ. Vascularized groin lymph node flap transfer for postmastectomy upper limb lymphedema: flap anatomy, recipient sites, and outcomes. Plast Reconstr Surg 2013;131:1286.

[42] Dancey A, Nassimizadeh A, Nassimizadeh M, Warner RM, Waters R. A chimeric vascularised groin lymph node flap and DIEP flap for the management of lymphoedema secondary to breast cancer. J Plast Reconstr Aesthet Surg 2013;66:735. 